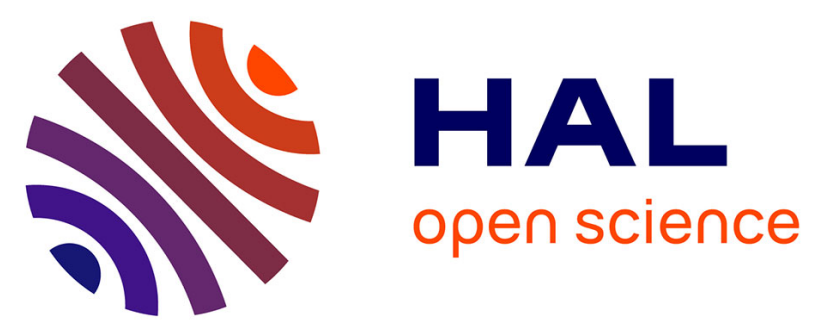

\title{
PATCH-BASED SAR IMAGE CLASSIFICATION: THE POTENTIAL OF MODELING THE STATISTICAL DISTRIBUTION OF PATCHES WITH GAUSSIAN MIXTURES
}

\author{
Sonia Tabti, Charles-Alban Deledalle, Loïc Denis, Florence Tupin
}

\section{To cite this version:}

Sonia Tabti, Charles-Alban Deledalle, Loïc Denis, Florence Tupin. PATCH-BASED SAR IMAGE CLASSIFICATION: THE POTENTIAL OF MODELING THE STATISTICAL DISTRIBUTION OF

PATCHES WITH GAUSSIAN MIXTURES. IGARSS, Jul 2015, Milan, Italy. hal-01158555

\section{HAL Id: hal-01158555 \\ https://hal.science/hal-01158555}

Submitted on 1 Jun 2015

HAL is a multi-disciplinary open access archive for the deposit and dissemination of scientific research documents, whether they are published or not. The documents may come from teaching and research institutions in France or abroad, or from public or private research centers.
L'archive ouverte pluridisciplinaire HAL, est destinée au dépôt et à la diffusion de documents scientifiques de niveau recherche, publiés ou non, émanant des établissements d'enseignement et de recherche français ou étrangers, des laboratoires publics ou privés. 


\title{
PATCH-BASED SAR IMAGE CLASSIFICATION: THE POTENTIAL OF MODELING THE STATISTICAL DISTRIBUTION OF PATCHES WITH GAUSSIAN MIXTURES
}

\author{
Sonia Tabti ${ }^{1}$, Charles-Alban Deledalle ${ }^{2}$, Lö̈c Denis ${ }^{3}$, Florence Tupin $^{1}$ \\ ${ }^{1}$ Institut Mines-Télécom, Télécom ParisTech CNRS-LTCI, Paris, France \\ ${ }^{2} \mathrm{CNRS}$-Univ. Bordeaux, IMB, Talence, France \\ ${ }^{3}$ CNRS-Univ. Saint-Etienne, Laboratoire Hubert Curien, Saint-Etienne, France
}

\begin{abstract}
Due to their coherent nature, SAR (Synthetic Aperture Radar) images are very different from optical satellite images and more difficult to interpret, especially because of speckle noise. Given the increasing amount of available SAR data, efficient image processing techniques are needed to ease the analysis. Classifying this type of images, i.e., selecting an adequate label for each pixel, is a challenging task. This paper describes a supervised classification method based on local features derived from a Gaussian mixture model (GMM) of the distribution of patches. First classification results are encouraging and suggest an interesting potential of the GMM model for SAR imaging.
\end{abstract} GMM.

Index Terms - SAR images, patches, classification,

\section{INTRODUCTION}

This work considers the image classification task in which a class (or label) is associated to each pixel of an image. It is an important research area in remote sensing imagery because of its many applications such as map updating or natural disaster assessment. Concerning Synthetic Aperture Radar (SAR) imagery, due to many sensors launched in the past years, many algorithms have been designed for different types of SAR data. Multi-sensor approaches deal both with an optical and a SAR amplitude image [1], while other approaches use polarimetric data [2]. In this paper we focus on the use of a single amplitude SAR image.

There are a lot of classification approaches inspired by different research fields. Some of them are based on machine learning techniques, such as random forests [3] or Support Vectors Machines (SVM) [2]. Other methods aim at modeling the amplitude and the texture of the image. In [4], the authors proposed to use a Nakagami distribution which is the standard choice to model an amplitude SAR image, whereas in [5], a Fisher distribution is chosen for its better ability to model urban areas. Voisin et al. proposed in [6] a dictionary

This work was partially funded by the DGA. of parametric families including log-normal, Weibull, Nakagami and Generalized Gamma distribution. GreyLevel Cooccurence Matrices (GLCM) are higher-order features often used to describe the texture since they better discriminate urban areas compared to a semi-variogram approach [6]. Texture can also be described by an auto-regression model as in [4], which helps improving the classification results when the image contains many textures.

To avoid a lack of spatial coherence on the classification map, regularization models are needed. Markovian modeling has been extensively used in this context [5] as well as hierarchical Markovian modeling starting from an oversegmentation obtained by a watershed algorithm [7] . The prior designed in [8] incorporates a conditional multinomial auto-logistic random field into a Normalized Gamma Process mixture. It allows a flexibility in the number of classes and gives smooth classification results.

In this paper, we propose a supervised amplitude SAR image classification algorithm with features based on a Gaussian Mixture Model (GMM) of the statistical distribution of patches. We study the ability of GMM learned on a huge database of patches [9] to describe the amplitude and the texture of SAR content with a bag-of-words-like approach. Section 2 introduces the followed GMM approach, section 3 describes the classification process and section 4 presents some results.

\section{GAUSSIAN MIXTURE MODELS BASED FEATURE}

Patches are small portions of images and they are used in many image processing tasks because they are easier to model in comparison with the whole image. GMM are known to well model the distribution of patches extracted from natural images. The Expected Patch Log-Likelihood (EPLL) algorithm introduced in [9] and extended to SAR imaging in [10] achieves state-of-the-art denoising performance. Its GMM is learned with an Expectation-Maximization algorithm applied to $10^{6}$ patches of size $8 \times 8$ extracted from natural images. The learnt GMM is made of $K=200$ zero-mean Gaussian components. A patch denoted by $\bar{x}$, whose mean has been 

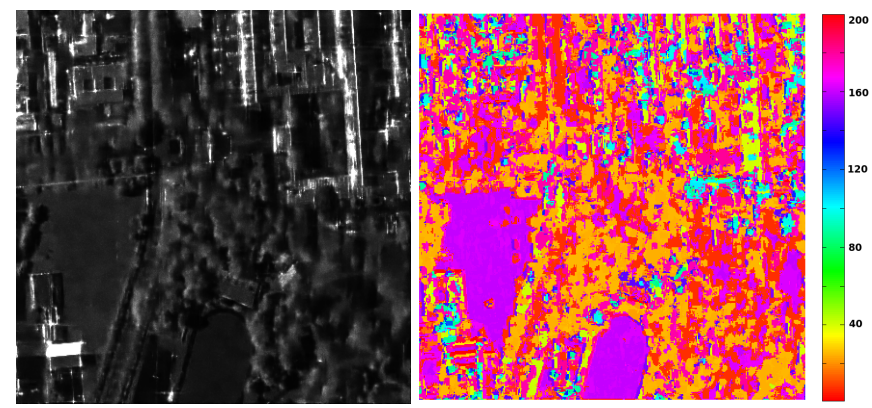

Fig. 1. The denoised version of a 1-look SAR image using [10] and the corresponding Gaussian models map. Each color of the colormap respresents one of the Gaussian models. These models have been ranked according to their similarity in a circular way using the Kullback-Leibeler divergence. One can see that the Gaussian models map represents well the structures observed in the SAR image.

previously subtracted, can be described with the following generative mixture model

$$
\mathrm{p}(\overline{\boldsymbol{x}})=\sum_{k=1}^{K} w_{k} n_{k} \exp \left\{-\frac{1}{2} \overline{\boldsymbol{x}}^{t} \boldsymbol{\Sigma}_{k}^{-1} \overline{\boldsymbol{x}}\right\}
$$

with $\Sigma_{k}$ the full-rank covariance matrix of the $k$-th model, $w_{k}$ the $k$-th mixweight such that $\sum_{k=1}^{K} w_{k}=1$ and $n_{k}=$ $\operatorname{det}\left(2 \pi \boldsymbol{\Sigma}_{k}\right)^{-1 / 2}$ a normalization constant. We denote by the $k$-th atom the quantity $\boldsymbol{a}_{k}=\left(\boldsymbol{\Sigma}_{k}, w_{k}\right)$ for $k \in\{1, \ldots, K\}$.

EPLL has been adapted to SAR image restoration in [10] where its performance for despeckling has been shown to be similar to the state of the art. As a by product, the algorithm provides a map of the best atom selected to represent each patch $\overline{\boldsymbol{x}}$ of the denoised image ${ }^{1}$ as expressed below

$$
\underset{k}{\operatorname{argmax}} w_{k} n_{k} \exp \left\{-\frac{1}{2} \overline{\boldsymbol{x}}^{t} \boldsymbol{\Sigma}_{k}^{-1} \overline{\boldsymbol{x}}\right\}
$$

As the model learned in [9] has proven being powerful enough to encode a large variability of structures within a patch (as shown by its efficiency at despeckling SAR images in [10]), it may provide useful features to represent local structures in SAR images see figure 1 . We propose in the following to assess the ability of this GMM based feature through a simple classification task.

\section{GMM-BASED CLASSIFICATION FRAMEWORK}

Our supervised classification framework is divided in two parts. The first one consists in learning the frequencies of each atom and each quantized radiometry in a given class. The second step classifies each pixel within a Bayesian

\footnotetext{
${ }^{1}$ The overlap used during the patch extraction implies that the number of patches is equal to the number of pixels so each pixel is represented by a Gaussian model.
}

framework based on its corresponding atom, radiometry and neighborhood.

\subsection{Learning the classification features}

To achieve the training procedure, we first denoise a set of eight amplitude-calibrated TerraSAR-X SAR images of one meter resolution using the procedure described in [10]. The sizes of the images are between $1000 \times 1000$ and $2000 \times 2000$ pixels, and they are manually labeled into $N_{\mathcal{L}}=3$ classes: urban area, high vegetation, homogeneous area (low vegetation and water). After this step, the corresponding map of atoms introduced in Sec. 2 is used to compute the frequency $\mathrm{P}\left(\boldsymbol{a}_{k} \mid \mathcal{L}\right)$ of each atom $\boldsymbol{a}_{k}, k \in\{1, \ldots, K\}$, for each label $\mathcal{L}$ in the set of $N_{\mathcal{L}}$ labels see figure.2.

Since the GMM-based feature modeling the structure of patches is obtained after a mean subtraction, it is necessary to also consider the radiometry of pixels as an extra feature. The data-set is thus quantized into $Q=10$ levels and the same process is applied to learn the frequency $\mathrm{P}\left(\boldsymbol{\nu}_{q} \mid \mathcal{L}\right)$ of each radiometry level $\boldsymbol{\nu}_{q}, q \in\{1, \ldots, Q\}$, for each label in the set $\mathcal{L}$.

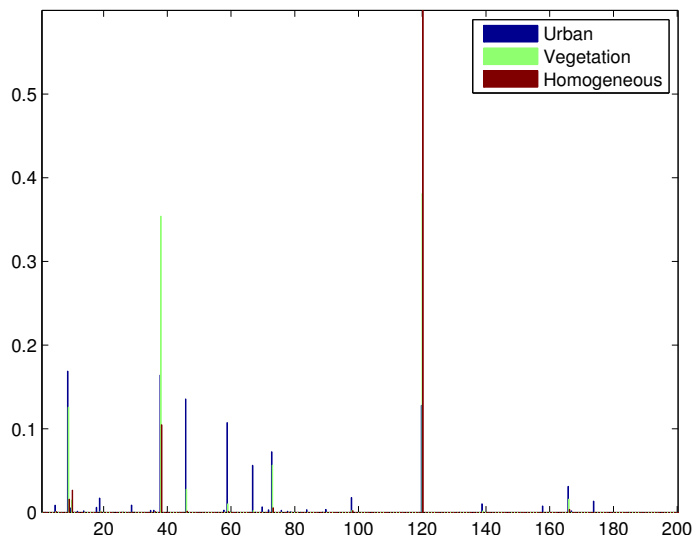

Fig. 2. Histograms representing the probability of occurence of each Gaussian Model for each label. Each histogram varies from one label to another and this allows the GMM-based feature to discriminate the different labels as we will see in paragraph 3.2.

\subsection{Label assignation}

The classification step has been performed on three amplitude TerraSAR-X SAR images given in Fig. 3. As for the training step, each pixel $i$ of the image of interest is represented by its corresponding atom $\boldsymbol{a}^{i}$ (obtained with EPLL) and its quantized radiometry $\boldsymbol{\nu}^{i}$. Given the frequency of each Gaussian model and each gray-level conditionally to the labels learned during the training step, see Sec. 3.1, a log-likelihood for each 
label $\mathcal{L}$ can be assigned to each pixel $i$ as follow

$$
\log \mathrm{P}\left(\boldsymbol{a}^{i}=\boldsymbol{a}_{k}, \boldsymbol{\nu}^{i}=\boldsymbol{\nu}_{q} \mid \mathcal{L}\right)=\log \mathrm{P}\left(\boldsymbol{a}_{k} \mid \mathcal{L}\right)+\log \mathrm{P}\left(\boldsymbol{\nu}_{q} \mid \mathcal{L}\right)
$$

assuming the radiometry of a patch is independent of its structure captured by its corresponding atom. This quantity represents the probability for a pixel $i$ to belong to the class of label $\mathcal{L}$. To enforce regularity and avoid a lack of spatial coherence on the classification map, we moreover assume that the class of a pixel of interest depends on the class affected to its neighboring pixels. By adopting a Bayesian framework jointly with a Potts regularization term, this leads to affect the label $\mathcal{L}^{i}$ to each pixel $i$ that maximizes the overall a posteriori probability, i.e., such that the following is minimized:

$$
-\log \sum_{i} \mathbf{P}\left(\boldsymbol{a}^{i}, \boldsymbol{\nu}^{i} \mid \mathcal{L}^{i}\right)+\beta \sum_{i \sim j} \delta\left(\mathcal{L}^{i}, \mathcal{L}^{j}\right)
$$

where $\beta>0, i \sim j$ refers to the indices of neighbor pixels in a eight-connectivity system and $\delta\left(\mathcal{L}^{i}, \mathcal{L}^{j}\right)=1$ if $\mathcal{L}^{i}=\mathcal{L}^{j}, 0$ otherwise. Satisfying solutions of such discrete optimization problems can be iteratively obtained by graph-cuts with the $\alpha-\beta$ swap strategy described in [11].

\section{RESULTS}

Classification results on the three testing images are given in Fig. 3 and show that GMM-based features perform well to capture the diversity of SAR content. Our preliminary results offer a promising classification error rate, above $75 \%$, with respect to a ground truth obtained by manually labeling this testing data-set.

\section{CONCLUSION}

Beyond the application of GMMs to speckle noise reduction [10], these models can form the core of image analysis tasks such as image classification. We proposed in this paper to learn the frequency of each component of the GMM within patches from 3 classes: urban area, vegetation and homogeneous area (low vegetation and water). This learning step is performed on a ground truth classification. The distribution of radiometry in each class is also captured. Then, classification is performed by fusing information from the Gaussian components, from the radiometry and a spatial coherency constraint. Promising results are obtained using these features. Further work includes refining these results by labeling a larger training data-set with more than three classes, using more sophisticated learning techniques, e.g., with SVM, and performing comparisons with state of the art methods.

\section{REFERENCES}

[1] A. Voisin, V. Krylov, G. Moser, S.B. Serpico, and J. Zerubia, "Supervised classification of multisensor and multiresolution remote sensing images with a hierarchical copula-based approach," IEEE Transactions on Geoscience and remote sensing, 2014.

[2] C. Lardeux, P-L. Frison, C. Tison, J-C. Souyris, B. Stoll, B. Fruneau, and J-P. Rudant, "Support vector machine for multifrequency SAR polarimetric data classification," IEEE Transactions on Geoscience and Remote Sensing, vol. 47, no. 12, pp. 4143-4152, 2009.

[3] W. Yang, T. Zou, D. Dai, and Y. Shuai, "Supervised land-cover classification of TerraSAR-X imagery over urban areas using extremely randomized clustering forests," in Urban Remote Sensing Event, 2009 Joint, May 2009, pp. 1-6.

[4] K. Kayabol and J. Zerubia, "Unsupervised amplitude and texture classification of SAR images with multinomial latent model," IEEE Transactions on Image Processing, vol. 22, no. 2, pp. 561-572, 2013.

[5] C. Tison, J-M. Nicolas, F. Tupin, and H. Maître, "A new statistical model for markovian classification of urban areas in high-resolution SAR images," IEEE Transactions on Geoscience and Remote Sensing, vol. 42, no. 10, pp. 2046-2057, 2004.

[6] A. Voisin, G. Moser, V. Krylov, S.B. Serpico, and J. Zerubia, "Classification of very high resolution SAR images of urban areas by dictionary-based mixture models, copulas and Markov random fields using textural features," in SPIE Remote Sensing, Lorenzo Bruzzone, Ed., Toulouse, France, Sept. 2010, vol. 7830, SPIE.

[7] Y. Yong, S. Hong, and H. Chu, "Supervised SAR image MPM segmentation based on region-based hierarchical model," Geoscience and Remote Sensing Letters, IEEE, vol. 3, no. 4, pp. 517-521, Oct 2006.

[8] K. Kayabol and B. Gunsel, "Unsupervised classification of SAR images using normalized gamma process mixtures," Digital Signal Processing, vol. 23, no. 5, pp. 1344-1352, 2013.

[9] D. Zoran and Y. Weiss, "From learning models of natural image patches to whole image restoration," ICCV, 2011.

[10] S. Tabti, C-A. Deledalle, L. Denis, and F. Tupin, "Modeling the distribution of patches with shift-invariance: application to SAR image restoration," ICIP, 2014.

[11] Y. Boykov, O. Veksler, and R. Zabih, "Fast approximate energy minimization via graph cuts," Pattern Analysis and Machine Intelligence, IEEE Transactions on, vol. 23, no. 11, pp. 1222-1239, 2001. 

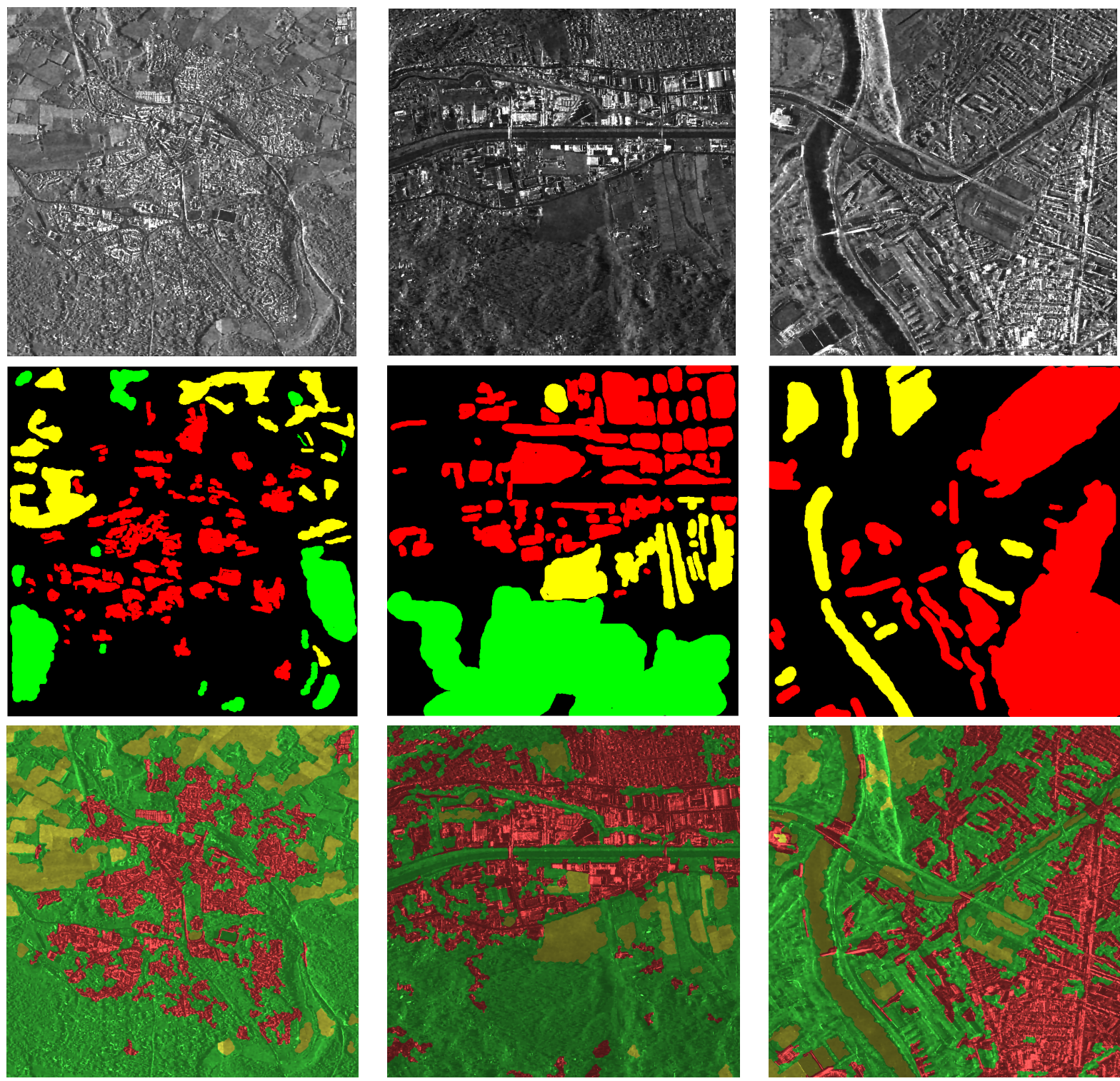

$91.71 \%$

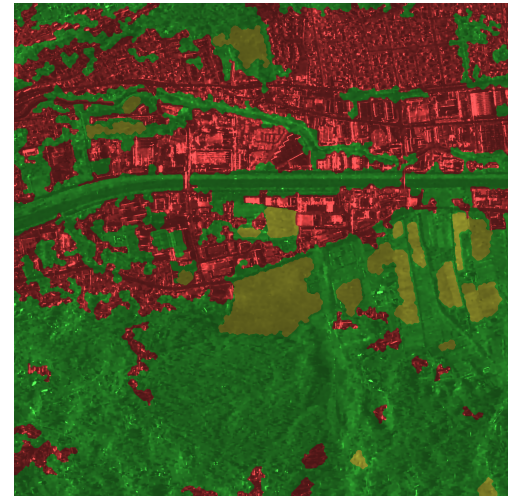

$86.71 \%$

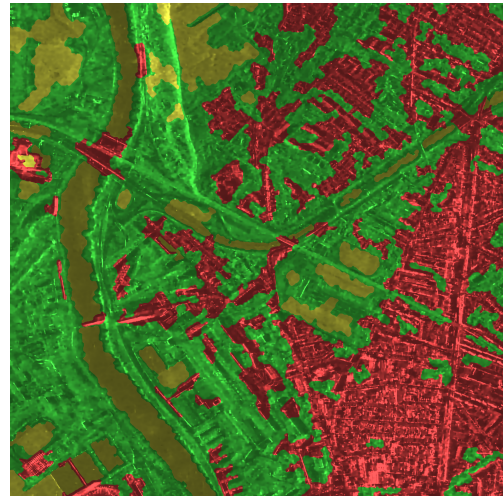

$75.37 \%$

Legend: Urban, High vegetation, Low vegetation and water, Unlabeled

Fig. 3. From first to last row: despeckled TerraSAR-X amplitude SAR images, ground truth, classification results and percentage of correctly classified pixels over the labeled pixels in the ground-truth. 